Gut, 1987, 28, 1625-1629

\title{
Significance of a fall in serum CEA concentration in patients treated with cytotoxic chemotherapy for disseminated colorectal cancer
}

\author{
T G ALLEN-MERSH, N KEMENY, D NIEDZWIECKI, B SHURGOT, AND \\ J M DALY
}

From the Departments of Surgery, Medical Oncology, and Biostatistics, Memorial Sloan-Kettering Cancer Center, New York, NY, USA.

SUMmARY 'Tumour response', defined as clinical or radiological evidence of tumour shrinkage is frequently regarded as an objective of chemotherapy, rather than as a predictor of prolonged survival. This study has assessed whether a fall in the serum CEA concentration after chemotherapy for disseminated colorectal cancer is a predictor of prolonged survival and compared it with tumour response as a predictor of survival. There was a $37 \%$ improvement in median survival among patients whose serum CEA concentration fell after chemotherapy $(70 \%$ of patients treated) compared with patients whose serum CEA did not fall. The use of $>25 \%$ clinical or radiological tumour shrinkage as a predictor of prolonged survival identified a smaller proportion $(36 \%)$ of patients in whom there was a $52 \%$ prolongation in median survival compared with patients whose tumours shrank less than $25 \%$, or did not shrink. Proportional hazards regression analysis suggested that tumour shrinkage was a stronger predictor of survival. A fall in serum CEA concentration, however, identified a group of patients whose tumours did not shrink, but who had a $27 \%$ improvement in median survival compared with those whose tumours did not shrink and whose serum CEA concentration did not fall. Monitoring of the serum CEA during the first two months of treatment appears to provide a sensitive and economical means of identifying those patients whose survival is likely to be prolonged by chemotherapy for colorectal cancer.

The value of serum carcinoembryonic antigen (CEA) monitoring in the detection of recurrent colorectal cancer has been established.' Some patients with recurrent disease can be treated by a second surgical resection but for most, cytotoxic chemotherapy offers the only chance of slowing the progress of the disease. The potential benefits of effective chemotherapy are a prolongation of survival and an improvement in performance status.

It would be useful to establish criteria which could identify early, those patients whose survival will be prolonged by chemotherapy. It is generally assumed that clinical or radiological evidence of tumour shrinkage, referred to as response, is associated with

Address for correspondence: Mr T G Allen-Mersh. FRCS. Department of Surgery, Charing Cross Hospital. Fulham Palace Road, London W6 8RF

Received for publication 8 May 1987. prolongation of survival. Among patients with serum CEA producing tumours, a change in the level of serum CEA may reflect a change in the burden of disease. Thus, a reduction in the serum CEA concentration after chemotherapy might also predict prolonged survival, as has been suggested in a study of 36 patients by Hine and Dykes."

The objective of this study was to determine whether a fall in serum CEA after chemotherapy was associated with prolonged survival in a population of over 300) patients treated with chemotherapy for disseminated colorectal cancer.

\section{Methods}

PATIENTS

The population under study consisted of 329 patients with metastatic colorectal carcinoma, who received 
chemotherapy at Memorial Sloan-Kettering Cancer Center (MSKCC) within the period 1976 to 1984. Two hundred and sixty five patients received systemic chemotherapy: 137 received methylCCNU, 5-fluorouracil, vincristine and streptozotocin (MOF-Strep); 22 received methotrexate and 5-fluorouracil; and 106 received methylCCNU, vincristine and 5-fluorouracil (MOF). Sixty four patients received hepatic infusional therapy with fluorodeoxyuridine (FUDR).

Response was defined in the following manner: a partial response (PR) was a $>50 \%$ reduction in the sum of the perpendicular axes of the tumour, measured radiologically for at least four weeks; a minor response (MR) denoted a $>25 \%$ but $<50 \%$ tumour reduction of measurable disease; stable disease was defined as no growth in tumour size for at least two months. Two serum CEA measurements were used, an initial value and the nadir which usually occurred within two months after the start of chemotherapy. A fall in serum CEA was defined as any reduction below the pretreatment serum CEA level. To allow for measurement error a more stringent definition of CEA fall as $>10 \%$ fall from baseline was also considered. All serum CEA measurements were done in the same laboratory, by an indirect method using Hansen's assay. ${ }^{3}$ Karnofsky performance status (KPS), serum lactic dehydrogenase (LDH), alkaline phosphatase (alk phos), and white blood count (WBC) were measured in all patients before treatment.

\section{STATISTICAL ANALYSIS}

Survival was measured from the time of protocol entry until death or last follow up. The survival distributions for different groups of patients were estimated using the Kaplan-Meier, or product limit method $^{+}$and then compared by the logrank test. ${ }^{5}$ Confidence limits (CL) for median survival times were determined using the method proposed by Simon and Lee. ${ }^{\circ}$ A consideration in these analyses was length bias because the chance of a patient being classified as a responder is influenced by the length of survival. As this study was retrospective in nature, the 'landmark method' of adjusting for length bias could not be used. ' To reduce the effect of length bias in survival analyses involving tumour shrinkage and change in CEA, patients who died within the first two months of receiving chemotherapy were omitted from the survival analyses. Thus there was a total of 290 patients in the sample ( $88 \%$ of total).

The relative importance of several predictors of survival was assessed by stepwise proportional hazards regression analysis. ${ }^{\text {}}$ The variables in the model were: CEA decrease $v$ CEA increase or no change in CEA; response $v$ no response; $\mathrm{LDH}<229$
$\mathrm{U} / 1 v \mathrm{LDH} 230-500 \mathrm{U} / 1 v \mathrm{LDH}>500 \mathrm{U} / \mathrm{l} ; \mathrm{WBC}$ $<10000$ cells $\mathrm{mm}^{3} v$ WBC $>10000$ cells $\mathrm{mm}^{2}$; alk phos $<115 \mathrm{U} / \mathrm{l} v$ alk phos $>115 \mathrm{U} / \mathrm{l}$; KPS $<70 v$ KPS $>70$; route of chemotherapy administration, regional $v$ systemic infusion.

The initial serum CEA concentrations in patients whose CEA decreased were compared with those in patients whose CEA remained the same or increased using Student's $t$ test. To control for variation in serum CEA concentrations, the natural logs of the serum CEA were taken when making comparisons.

\section{Results}

TUMOUR SHRINKAGE AS A PREDICTOR OF SURVIVAL

One hundred and nine patients had a tumour response which was classified as a PR or MR. The median survival of these patients was 13.7 months (95\% CL $12 \cdot 0-17 \cdot 7$ months) which was significantly $(\mathrm{p}<0.00001)$ better than the median survival of nine months (CL 8.2-10.5 months) in the remaining patients who did not have a documented response (Fig. 1).

CHANGE IN SERUM CEA CONCENTRATION AS A PREDICTOR OF SURVIVAL

The serum CEA fell in $70 \%$ of patients usually within two months of starting chemotherapy. Sixty two per

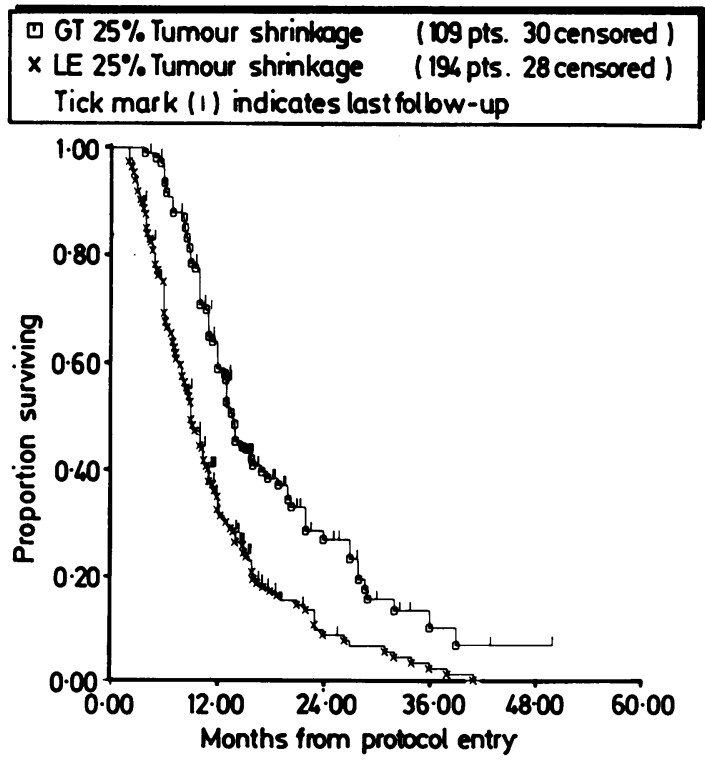

Fig. 1 There was a significant $(p<0.00001)$ improvement in the survival of patients whose tumours decreased $>25 \%$ after chemotherapy ( $\square$ ) compared with patients whose tumours decreased by $<25 \%$ or did not change $(X)$. 
cent of these patients in whom the serum CEA fell experienced a fall of $>20 \%$ of the initial serum CEA value. The serum CEA remained stable or continued to rise in the remaining $30 \%$ of the patients. Among the patients in whom the serum CEA fell after chemotherapy median survival was 12 months $(\mathrm{CL}$ $11.0-13.7$ months $)$ which was significantly $(p=0.0002)$ longer than a median survival of 8.8 months (CL 6.710.5 months) for those with no fall in serum CEA (Fig. 2). Allowing for an error in the measurement of serum CEA decrease, patients in whom serum CEA fell $>10 \%$ from baseline after chemotherapy were compared with those with no fall, stable values or a $<10 \%$ fall. Those with a $>10 \%$ fall in CEA had a significantly longer survival $(p=0.0002)$. There was no significant difference between the two groups in the pretreatment serum CEA level. The geometric means of the natural logarithms of pretreatment serum CEA were compared because of large variability in the raw values (a mean of $495.9 \pm 1677$ and $432 \cdot 68 \pm 3493$, for patients with no serum CEA fall versus those with a CEA fall, respectively). The numbers of patients receiving each treatment regimen were comparably distributed between those patients experiencing a fall in CEA and those who did not $(p=0 \cdot 16)$.

\section{RELATIONSHIP BETWEEN CHANGE IN SERUM} CEA CONCENTRATION AND SURVIVAL AMONG NON-RESPONDERS

Among the 175 patients who did not show a documented tumour response, patients who had a serum

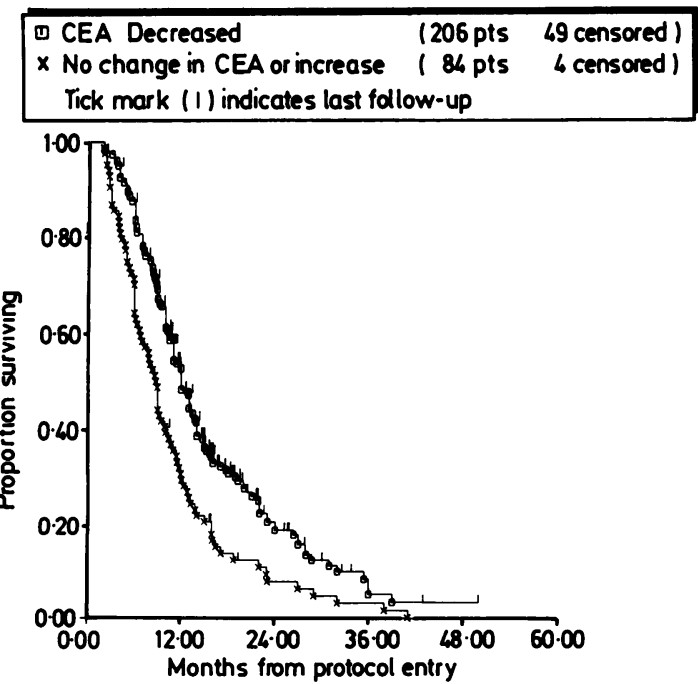

Fig. 2 There was a significant $(p<0.0002)$ improvement in the survival of patients whose serum CEA concentration fell after chemotherapy ( $\square$ ) compared with patients in whom $C E A$ did not fall $(X)$.

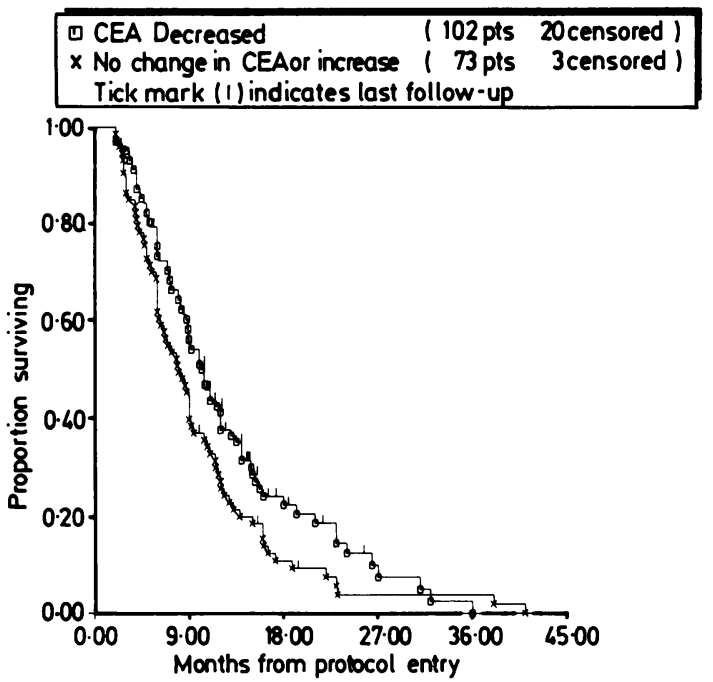

Fig. 3 Among patients in whom tumour shrinkage of $>25 \%$ did not occur (non-responders) there was a significant $(p=0.04)$ improvement in survival among those in whom the serum $C E A$ concentration fell $(\square)$ compared with those in whom $C E A$ did not fall $(X)$.

CEA decrease experienced significantly $(p=0 \cdot 04)$ longer survival than patients whose CEA rose or remained the same (Fig. 3). The estimated median survival in non-responders whose serum CEA fell was $10 \cdot 2$ months (CL 9.0-12.0 months) versus $8 \cdot 0$ months (CL 6.1-9.0 months) for the remaining nonresponders with no fall in serum CEA.

Univariate survival analyses indicated that KPS, LDH, alk phos, and type of treatment were significantly related to survival (Table 1). Alkaline phosphatase, however, was not included in the final model because it was not significant in the multivariate analysis. Allowing for initial KPS, serum LDH and type of treatment, a fall in the serum CEA remained a significant predictor of improved survival (improvement $\chi^{2} p=0 \cdot 001$ ). In addition a fall in serum CEA remained predictive of survival even when eventual response status was considered in the analysis (improvement $\chi^{2} p=0 \cdot 008$ ).

STABLE DISEASE SUBGROUP

In sixty three patients the serum CEA concentration did not increase or decrease more than $20 \%$ from pretreatment baseline. There was no significant difference $(p=0.4)$ between the survival curves of patients whose CEA concentration fell by $<20 \%$, median survival 12.0 months (CL 6.0 -11.5 months) compared with patients whose CEA level rose by $<20 \%$ or remained the same, median survival 8.6 months (CL 6.0-11.5 months). With this sample size the power to detect a difference is $60 \%$. $^{9}$ 
Table 1 Pretreatment factors which were shown on univariate analysis to influence survival

\begin{tabular}{lc}
\hline Variable & Value \\
\hline Karnofsky performance status & $<0 \cdot()(0)(0) 1$ \\
Serum lactic dehydrogenase & $<0 \cdot()(0)(0) 1$ \\
Alkaline phosphatase & $0 \cdot(0)(0) 1$ \\
Type of treatment & $(0 \cdot()(0) 1$ \\
\hline
\end{tabular}

\section{Discussion}

Predictors of improved survival resulting from chemotherapy can be viewed as a continuum from the most rigorous - that is, complete tumour disappearance, through varying degrees of tumour shrinkage, to a fall in the serum CEA concentration without tumour shrinkage. Thus response as defined by tumour shrinkage is a more stringent criterion than response as defined by a fall in the serum CEA concentration and may predict a different enhancement in survival. For this reason when comparing chemotherapy studies, a fall in the serum CEA concentration should not be equated with other criteria of response. Previous studies (Table 2), treating tumour shrinkage as an objective or endpoint of treatment, have suggested that a decrease in serum CEA is a poor predictor of tumour shrinkage because while it is sensitive (low false negative) it is also nonspecific (high false positive).

Rather than regarding tumour shrinkage as an objective of treatment, another approach is to consider survival as the endpoint of analysis and to seek to identify predictors of improved survival arising from chemotherapy. The results of our study indicate that a fall in the serum CEA concentration after chemotherapy, although not an accurate reflection of eventual tumour shrinkage, did identify patients whose survival was enhanced by chemotherapy. The estimated median survival for patients who experienced a fall in serum CEA was $12 \cdot 0$ months. If this group was characterised further as those experiencing a CEA reduction of $<20 \%$,

Table 2 Relationship between tumour responses and fall in $C E A$. A fall in serum CEA frequently occurs in the absence (false +ve) of response-defined as tumour shrinkage

\begin{tabular}{|c|c|c|c|c|}
\hline \multirow[b]{2}{*}{ Reference } & \multirow[b]{2}{*}{$n$} & \multirow[b]{2}{*}{ Response \% } & \multicolumn{2}{|c|}{$\begin{array}{c}\text { Prediction of response by CEA } \\
\text { test }\end{array}$} \\
\hline & & & False + & False - \\
\hline 10 & 93 & 10 & $17 / 26(65 \%)$ & $0 / 67 \quad(0 \%)$ \\
\hline 11 & 122 & 25 & $19 / 33(57 \%)$ & $17 / 98(19 \%)$ \\
\hline 12 & 24 & 17 & $4 / 8 \quad(50 \%)$ & $0 / 16 \quad(0 \%)$ \\
\hline 13 & 49 & 37 & $4 / 20(20 \%)$ & $2 / 29 \quad(7 \%)$ \\
\hline Total & 288 & $22 \%$ & $44 / 87(51 \%)$ & $19 / 201 \quad(9 \%)$ \\
\hline
\end{tabular}

$21-49 \%$ or $>50 \%$, the median survivals were $12 \cdot 0$, $10 \cdot 4$ and 12.9 months, respectively. This suggests that a fall in the serum CEA was more important than the size of the fall. This survival benefit occurred even when favourable pretreatment prognostic indicators such as high performance status and low LDH level were considered. Moreover, knowledge of serum CEA change provided additional information about survival when the eventual degree of tumour shrinkage was known. Thus, there was a component of change in serum CEA which related to survival and was not attributable to either pretreatment predictors $^{14}$ or to the propensity of the tumour to shrink after chemotherapy.

It has been suggested ${ }^{15}$ that, because of daily fluctuations in the serum CEA concentration, patients whose serum CEA remains within $\pm 32 \%$ of the previous baseline level form a homogenous subgroup who have stable disease. In our patients, the estimated median survival for patients whose CEA increased by $20 \%$ was less than the median survival for patients whose serum CEA fell by $20 \%$. As the power of this analysis was only $60 \%$, differences were not easily detectable; however, the estimated median survival times ( 12 and 8.6 months, respectively) were not clearly supportive of a homogenous subgroup of patients. In this study, where the serum CEA was measured before treatment and during the first two months of treatment, day to day fluctuations in serum CEA did not seem to obscure the improved survival associated with even a small $(>10 \%)$ fall in serum CEA.

The important consideration resulting from this investigation is that knowledge of a change in serum CEA concentration may be useful as an indicator of survival after chemotherapy. Both tumour shrinkage and reduction in serum CEA concentration appear to be useful in predicting prolonged survival in patients receiving cancer chemotherapy. Monitoring serum CEA values during treatment may be the more economical means of identifying those patients whose survival is likely to be prolonged by chemotherapy.

This study was presented at the British Society of Gastroenterology Meeting, September 1986. Abstract of which appeared in Gut 1986; 27: A1258.

\section{References}

1 NIH Consensus Statement. Carcinoembryonic antigen: its role as a marker in the management of cancer. Br Med J 1981; 282: 373-5.

2 Hine KR, Dykes PW. A prospective randomised trial of early cytotoxic therapy for recurrent colorectal cancer detected by serum CEA. Gut 1984; 25 : 682-8.

3 Hanson HJ, Syder LJ, Miller E, et al. Carcinoembryonic antigen (CEA) assay: a laboratory adjunct in the 
diagnosis and management of cancer. Hum Pathol 1974; 5: 139-47.

4 Kaplan EL. Meier P. Non parametric estimation from incomplete observations. J Am Statist Assoc 1958; 53: 457-81.

5 Elandt-Johnson RC, Johnson NL. Survival models and data analysis. New York: J Wiley and Sons, 1980.

6 Simon R, Lee YG. Non parameters confidence limits for survival probabilities and median survival time. Cancer Treat Rep 1982; 66: 37-42.

7 Anderson JR, Cain KC, Gelber RD. 'Analysis of survival by tumor response'. J Clin Oncol 1983; 1: 710-9.

8 Cox DR. Regression models and life tables. $J R$ Statist Soc (B) 1972; 34: 187-200.

9 Lesser ML, Canto SJ. Tables of power for the F-test for comparing two exponential survival distributions. J Chronic Dis 1983; 34: 533-44.

10 Herrera MA, Chu TM, Holyoke ED, Mittleman A. CEA monitoring of palliative treatment for colorectal carcinoma. Ann Surg 1977; 185: 23-30.
11 Shani I, O'Connel MJ, Moertel CG, Schutt AJ, Silvers A, Go VL. Serial plasma carcinoembryonic antigen measurements in the management of metastatic colorectal carcinoma. Ann Intern Med 1978; 88: 727-30.

12 Mayer RJ, Garnick MB, Steele GD, Zamcheck N. Carcinoembryonic antigen (CEA) as a monitor of chemotherapy in disseminated colorectal cancer. Cancer 1978; 42: 1428-33.

13 Al-Sarrat M, Baker L, Tulley R, Kithier K, Vaitkevicius VK. The value of serial carcinoembryonic antigen (CEA) in predicting response rate and survival of patients with gastrointestinal cancer treated with chemotherapy. Cancer 1979; 44: 1222-5.

14 Kemeny N, Braun DW. Prognostic factors in advanced colorectal carcinoma: the importance of lactic dehydrogenase, performance status and white blood cell count. Am J Med 1983; 74: 786-97.

15 Lokich JJ, Ellenberg S, Gerson B. Criteria for monitoring carcinoembryonic antigen: variability of sequential assays at elevated levels. J Clin Oncol 1984; 2; 181-6. 